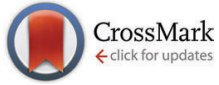

Cite this: Chem. Commun., 2016, 52,6131

Received 31st March 2016 Accepted 7th April 2016

DOI: $10.1039 / \mathrm{c} 6 \mathrm{cc} 02731 \mathrm{~h}$

www.rsc.org/chemcomm

\title{
Controlling microenvironments and modifying anion binding selectivities using core functionalised hyperbranched polymers $\dagger$
}

\author{
Georgia Mann, ${ }^{a}$ Lance J. Twyman*a and Philip A. Gale ${ }^{b}$
}

\begin{abstract}
An isophthalamide anion binding site has been incorporated into hyperbranched polymers resulting in a change in the selectivity of the receptor from chloride to bromide.
\end{abstract}

Isophthalamides have been explored as hydrogen bond donating receptors for anions ${ }^{1}$ and have been employed in a number of anion sensor ${ }^{2}$ and transporter ${ }^{3}$ applications. Embedding supramolecular receptors within hyperbranched polymers should provide a microenvironment around the binding site that will modulate the binding properties of receptor. Highly branched globular macromolecules possess architectures similar to the three dimensional/tertiary structure of proteins. ${ }^{4}$ Hyperbranched polymers (HBPs) can be synthesised relatively easily and techniques exist to incorporate binding sites within the internal domain of the globular structure. ${ }^{5}$ Such an approach allows precise control of both the nature of the binding site, and the microenvironment around it. Specifically, the hyperbranched polymer can effect both the steric and electronic properties of the binding site. ${ }^{6}$ As such, these functionalised synthetic macromolecules can mimic the controlled internal space and therefore the binding environment found within bio-macromolecules. $\$$

Results from previous work using core functionalized HBPs has shown that binding affinities are initially improved as the polymers molecular weight/size increases. ${ }^{7}$ This is due to the microenvironment provided by the polymeric structure. This increase in binding occurred until the molecular weight exceeded 6000-8000 Da. When molecular weights exceeded this value, there was a sharp decrease in the binding affinity. This property is also observed in dendrimers ${ }^{8}$ and the point at which this occurs is referred to as the dense packed/shell limit. ${ }^{9}$ Therefore we planned to use the same hyperbranched polymeric system, that utilizes the monomer 3,5-diacetoxybenzoic acid,

\footnotetext{
${ }^{a}$ Department of Chemistry, University of Sheffield, Brook Hill, Sheffield, S2 7HF, UK

${ }^{b}$ Chemistry, University of Southampton, Highfield, Southampton, SO17 1BJ, UK

$\dagger$ Electronic supplementary information (ESI) available: Synthetic details and titration data. See DOI: 10.1039/c6cc02731h
}

and aim for polymers with molecular weights below and above the previously observed dense packed limit. This can be done with relatively good control by varying the core to monomer ratio. ${ }^{10}$ In this communication we report the anion binding properties of an isophthalamide ${ }^{11}$ core unit within a hyperbranched polymer (formed from 3,5-diacetoxybenzoic acid) and compare the binding to a simple isophthalamide control molecule independent of hyperbranched polymer. Specifically, we describe how the binding affinities for a series of halides were reversed (with respect to the hard and soft character of the anion), when compared to those measured using the simple isophthalamide control ligand.

The isophthalamide selected for our study possessed 2 acyl units that would initiate polymerisation of the hyperbranched polymers via a trans-esterification process. The acylated core selected would also be used as the ligand for our control studies. As such, compound 1 was prepared by reaction of isophthaloyl chloride with 2 equivalents of 3 -aminophenol in $\mathrm{DMF}$ in the presence of $\mathrm{Et}_{3} \mathrm{~N}$. The intermediate isophthalamide was obtained in $64 \%$ yield after purification.

This compound was acetylated by reaction with an excess of acetyl chloride in pyridine. After removal of pyridine via vacuum distillation, the crude product was purified by recrystallization from dichloromethane and petroleum ether, to give the final acetylated isophalamide 1 in $65 \%$ yield, Scheme 1.

We decided to synthesise two polymers containing the isophthalamide core; one above the dense packed limit and a second smaller polymer that fell below it. This would allow us to explore the specific electronic environment around the host anion binding core, in addition to steric effects. The hyperbranched polymers were synthesised using a core ratio of $1: 5$ and $1: 20$, for the small and large hyperbranched polymers respectively. Both reactions were carried out using a modified version of the procedure previously reported. ${ }^{7,12} \mathrm{~N}, \mathrm{~N}$-bis(4-acetoxyphenyl)isophthalamide was added to the monomer 3,5-diacetoxybenzoic acid (in a 1:20 or 1:5 mole ratio). These were then heated with an equal weight of diphenyl at $225{ }^{\circ} \mathrm{C}$ for a period of 45 minutes. The temperature was then lowered to 


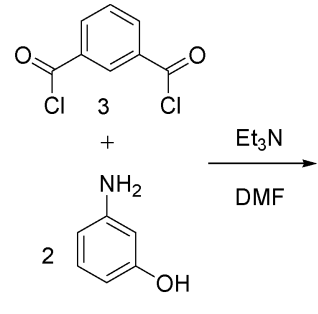

2
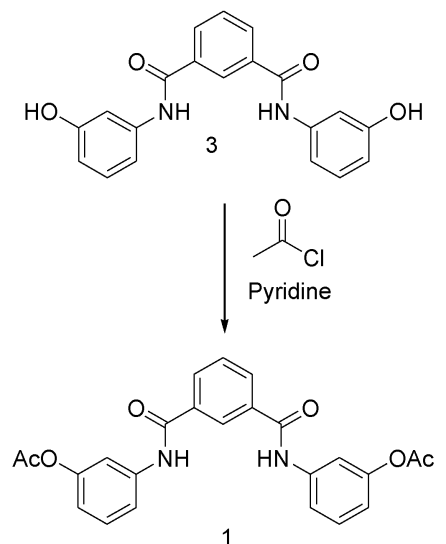

Scheme 1 Synthesis of the reference and polymer core compound 1.

$180{ }^{\circ} \mathrm{C}$ and the system was placed under a low vacuum for a period of 4 hours. This allowed removal of the acetic acid by-product and steered the reaction to completion with the formation of a glass-like solid. The use of reversible chemistry ensures that the core is integrated with a very high level of incorporation (approaching $100 \%$ - see ref. $5 a$ ). The final products were obtained by precipitation into methanol from the minimum amount of (warm) THF. The procedure was repeated twice more and the white solids formed washed thoroughly with cold methanol and dried prior to $71 \%$ yield respectively. A representative structure is shown in Scheme 2.

The stability constants for compound 1 with a series of halides added as their tetrabutylammonium salts were determined by
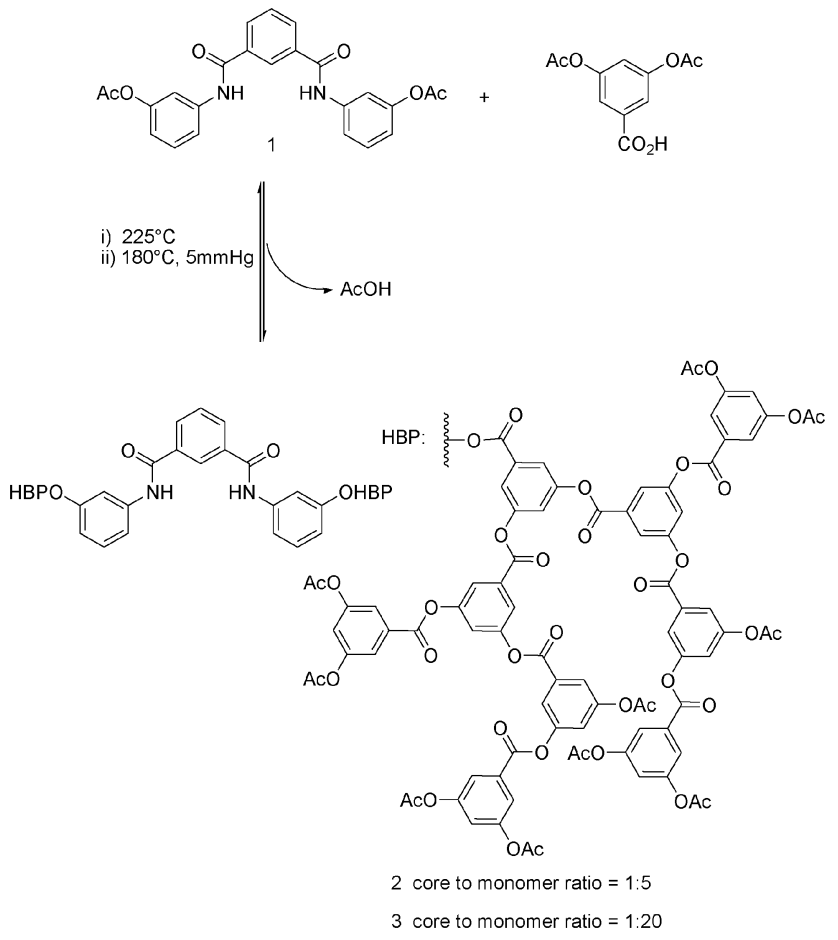

Scheme 2 Synthesis of the hyperbranched polymers 2 and $\mathbf{3}$ (with core to monomer ratios of $1: 5$ and $1: 20$ respectively).
Table 1 Stability constants for the anionic complexes of compound 1 and $\mathrm{HBPs} \mathbf{2}$ and $\mathbf{3}$ measured at $25^{\circ} \mathrm{C}$ in $\mathrm{CDCl}_{3}{ }^{a}$

\begin{tabular}{lcrr}
\hline Anion & Compound 1 $K_{\mathrm{a}}\left(\mathrm{M}^{-1}\right)$ & HBP 2 $\left(\mathrm{M}^{-1}\right)$ & HBP 3 $\left(\mathrm{M}^{-1}\right)$ \\
\hline $\mathrm{F}^{-}$ & $8900( \pm 18 \%)$ & $1900( \pm 15 \%)$ & $2300( \pm 15 \%)$ \\
$\mathrm{Cl}^{-}$ & $>10^{4}( \pm 15 \%)$ & $1700( \pm 21 \%)$ & $1200( \pm 18 \%)$ \\
$\mathrm{Br}^{-}$ & $5900( \pm 15 \%)$ & $10500( \pm 22 \%)$ & $11000( \pm 16 \%)$ \\
$\mathrm{I}^{-}$ & $800( \pm 15 \%)$ & $3650( \pm 15 \%)$ & $1400( \pm 15 \%)$ \\
${ }^{a}$ Errors shown in brackets. & & \\
\hline
\end{tabular}

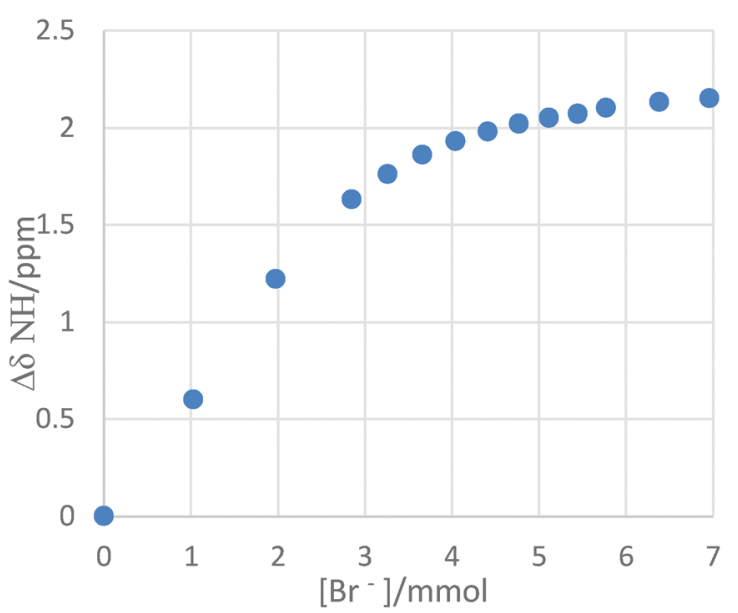

Fig. 1 Titration plot showing the change in NH shift position for the larger HBP 3 (5 mmol) and bromide (as the tetrabutylammonium salt).

${ }^{1} \mathrm{H}$ NMR titration techniques ${ }^{13}$ in $\mathrm{CDCl}_{3} . \S$ Job plot analysis was used to determine the stoichiometry of the complexes formed which was found to be $1: 1 .^{14}$ The results are shown in Table 1. Job plots and ${ }^{1} \mathrm{H}$ NMR titrations were repeated with hyperbranched polymers 2 and 3. The tritration plot for the larger HBP 3 and bromide (as the tetrabutylammonium salt) is shown in Fig. 1. It was found that $1: 1$ complexes with halides were formed in all cases. As we increased the steric bulk around the receptor, we noticed a drop in the binding affinities of fluoride and chloride. Specifically, the affinity dropped as we moved from receptor 1 to hyperbranched polymers 2 and 3 respectively (for each anion). Interestingly, we observed that although there is a drop in affinity, there is little difference between the affinities of HBPs 2 and 3, despite the increased steric bulk. This is presumably due to the relatively small size of the fluoride and chloride guests. On the other hand, the larger bromide behaves very differently and there is a significant enhancement in binding with this anion, as we go from compound 1 to HBPs 2 and 3 . As such, both HBPs are selective for bromide, Table 1 .

We also observed that the affinity for iodide is highest in HBP 2 followed by HBP 3 and then compound 1. Presumably, for both bromide and iodide the larger anions can interact with the polymer's $\pi$ system. ${ }^{15}$ This $\pi$-aromatic interaction is in addition to the hydrogen bonding, as evident from the $\mathrm{NH}$ (and $\mathrm{Ar}-\mathrm{H}$ ) shifts recorded during the NMR titrations. Therefore, the hydrogen bonding and $\pi$-aromatic interactions are working cooperatively to generate a higher binding constant for the larger halide anions; this is despite the obvious steric interactions. 
In addition to obtaining higher binding constants for the larger anions, we also noticed that the relative binding with respect to hard (fluoride and chloride) and soft (bromide and iodide) anions was effectively reversed. That is, although the order is not completely reversed with respect to each anion studied, binding does separate into two distinct groups. For example, the anions bound to the control ligand $\mathbf{1}$ in the following order; harder anions (chloride/fluoride) binding more strongly than the softer anions (bromide/iodide). In this respect the order appears to be related to the Hofmeister series. ${ }^{16}$ However, when hyperbranched polymers 2 and 3 were used, the order switches to the harder anions (chloride/ fluoride) (bromide/Iodide) binding less strongly than softer anions (bromide/Iodide). This change in selectivity is also attributed to local environment generated by the hyperbranched polymer (around the binding site). Specifically, cooperative $\pi$-interactions ${ }^{15}$ supporting the hydrogen bonding from the core.

By embedding an isophthalamide unit within a hyperbranched polymer it is possible to shift the selectivity of the binding site towards larger charge diffuse halides. We have concluded that this enhanced binding is due to a combination of hydrogen bonding and $\pi$-aromatic interactions working cooperatively to generate higher binding constants. This interesting finding demonstrates that hyperbranched polymer based receptors may have unique properties that are largely unexplored. Overall, the selectivity trend for the polymers shows that bromide binds strongest and the chloride the weakest. Effectively, the trend is reversed when compared to the reference isophthalamide receptor $\mathbf{1}$, which binds chloride the strongest and iodide the weakest. This therefore confirms that the microenvironment around a specific binding site/receptor can have a large effect on its binding potential and selectivity. Work is progressing in our laboratories to further study and exploit these properties, as well as studying the effect of structure and environment on the binding properties of other anions. In addition, we also plan to extend the study using alternative solvents that may direct or exclude binding at the polymeric isophthalamide core.

We acknowledge the EPSRC for funding (GM). We would also like to thank Professor Christopher Hunter (University of Cambridge, Department of Chemistry) for providing the software for the binding and Job plot analysis. PAG thanks the Royal Society and the Wolfson Foundation for a Research Merit Award.

\section{Notes and references}

\$ HBPs with anion binding groups at their periphery have been reported and show interesting binding related to polyvalence effects.
Initially binding involves two receptors bindings a single anion with $1: 1$ binding being observed as anion concentration increases. However, the receptors are not within the polymeric structure and changes in binding behaviour related to controlled microenvironments do not occur. $^{17}$

§ A typical titration was carried out using a standard NMR titration technique. Samples of host solution were prepared with a known concentration in $\mathrm{CHCl}_{3}(1-10 \mathrm{mM})$. Guest solutions were prepared in $\mathrm{CDCl}_{3}$ with concentrations ranging from 0.1-1.0 M. Each titration began with $0.7 \mathrm{~mL}$ of the host species in an NMR tube. The anionic guest was added in aliquots $(10-25 \mu \mathrm{L})$ to the host. The fitting software used took account of dilution. NMR scans (32) were run following each addition and the position of the NH chemical shift (ppm) was monitored. Solutions were made up fresh and used immediately after preparation. Anionic guests were added as tetrabutylammonium salts. Topspin 2.6 software was used to process the NMR spectra generated. Changes in chemical shift were fitted to a $1: 1$ binding isotherm in Microsoft Excel program. Experiments were repeated at least 3 times and the biding constant calculated from each fit. For each anion, the data presented herein is an average of the 3 binding constants.

1 K. Kavallieratos, C. M. Bertao and R. H. Crabtree, J. Org. Chem., 1999, 64, 1675-1683.

2 M. Más-Montoya, M. Cuartero, D. Curiel, J. A. Ortuño, M. S. García and A. Tárraga, Analyst, 2015, 140, 287-294.

3 P. V. Santacroce, J. T. Davis, M. E. light, P. A. Gale, J. C. IglesiasSánchez, P. Prados and R. Quesada, J. Am. Chem. Soc., 2007, 129, 1886-1887.

4 (a) K. Tahara, H. Shimakoshi, A. Tanaka and Y. Hisaeda, Dalton Trans., 2010, 3035-3042; (b) K. Kirkorian, A. Ellis and L. J. Twyman, Chem. Soc. Rev., 2012, 41, 6138-6159.

5 (a) P. J. Gittins, J. Alston, Y. Ge and L. J. Twyman, Macromolecules, 2004, 37, 7428; (b) Y. Ge and L. J. Twyman, Chem. Commun., 2006, $1658-1660$.

6 (a) I. N. Kurniasih, J. Keilitz and R. Haag, Chem. Soc. Rev., 2015, 44, 4145-4164; (b) X. Zheng, I. R. Oviedo and L. J. Twyman, Macromolecules, 2008, 41, 7776-7779.

7 A. Ellis and L. J. Twyman, Macromolecules, 2013, 46(17), 7055-7061.

8 (a) B. Zhang, H. Yu, A. D. Schlüter, A. Halperin and M. Kröger, Nat. Commun., 2013, 4, 1993; (b) M. Wind, K. Saalwächter, U.-M. Wiesler, K. Müllen and H. W. Spiess, Macromolecules, 2002, 35(27), 10071-10086; (c) A. Ellis, M. Wallace and L. J. Twyman, Chem. Commun., 2013, 49(73), 8063-8065; (d) J. Burnett, A. S. H. King and L. J. Twyman, React. Funct. Polym., 2006, 66(1), 187-194.

9 P.-G. de Gennes and H. Hervet, J. Phys., 1983, 44, 351.

10 P. J. Gittins and L. J. Twyman, J. Am. Chem. Soc., 2005, 127, 1646-1648.

11 (a) P. A. Gale, Acc. Chem. Res., 2006, 39, 465-475; (b) P. A. Gale, J. Garric, M. E. Light, B. A. McNally and B. D. Smith, Chem. Commun., 2007, 1736-1738.

12 S. R. Turner, B. I. Voit and T. H. Mourey, Macromolecules, 1993, 26, 4617-4622.

13 P. Thordarson, Chem. Soc. Rev., 2011, 40, 1305-1323.

$14 \mathrm{H}$. J. Schneider and A. Tatsimirsky, Principles and methods in supramolecular chemistry, Wiley, Chichester, 2000.

15 D. X. Wang and M. X. Wang, J. Am. Chem. Soc., 2013, 135, 892-897.

16 F. Hofmeister, Arch. Exp. Pathol. Pharmakol., 1888, 24, 247.

17 Q. Tan, L. Wang, L. Ma, H. Yu, Q. Liu and A. Xiao, Macromolecules, 2009, 42(13), 4500-4510. 